\title{
Solutions of Volterra integral and integro-differential equations using modified Laplace Adomian decomposition method
}

\author{
D. RANI AND V. MISHRA
}

\begin{abstract}
In this paper, an effectual and new modification in Laplace Adomian decomposition method based on Bernstein polynomials is proposed to find the solution of nonlinear Volterra integral and integro-differential equations. The performance and capability of the proposed idea is endorsed by comparing the exact and approximate solutions for three different examples on Volterra integral, integro-differential equations of the first and second kinds. The results shown through tables and figures demonstrate the accuracy of our method. It is concluded here that the non orthogonal polynomials can also be used for Laplace Adomian decomposition method. In addition, convergence analysis of the modified technique is also presented.
\end{abstract}

Mathematics Subject Classification 2010: 41A10; 44A10; 34A34; 45D05;65R10.

Keywords: Laplace transformation, Adomian decomposition method, modified Laplace Adomian decomposition method, Bernstein polynomials, Volterra integral and integro-differential equations.

\section{INTRODUCTION}

Substantial interest is devoted to solve nonlinear Volterra integral and integro-differential equations by many researchers and scientists due to its applications in science such as the population dynamics, spread of epidemics, semi-conductor devices [Wazwaz 2011], biological species coexisting together with increasing and decreasing rates of generating and in engineering such as heat transfer and neutron diffusion process [Bahuguna et al. 2009].

The nonlinear Volterra integral equation of the second kind is defined as [Wazwaz 2011]

$$
u(x)=f(x)+\int_{0}^{x} k(x, t) F(u(t)) d t
$$

where $f(x)$ is known as source term and $F$ is a nonlinear operator, $F(u(x))$ is a nonlinear function.

The nonlinear Volterra integro-differential equation of the first kind is given by 
[Wazwaz 2011; 2010]

$$
\int_{0}^{x} K_{1}(x, t) F(u(t)) d t+\int_{0}^{x} K_{2}(x, t) u^{(i)}(t) d t=f(x)
$$

However, the nonlinear Volterra integro-differential equation of the second kind is defined as [Wazwaz 2011; 2010]

$$
u^{(i)}(x)=f(x)+\int_{0}^{x} k(x, t) F(u(t)) d t
$$

where $u^{(i)}(x)$ denotes the ith order derivative of $u(x)$. The kernel $k(x, t)$ and the function $f(x)$ of these equations are given real-valued functions and $F(u(x))$ is a nonlinear function.

Earlier many numerical and analytical methods have been presented to solve these kinds of equations [Wazwaz 2011; 2010; Maleknejad and Najafi 2011; Maleknejad et al. 2011].

\subsection{Laplace Adomian decomposition method and modifications}

In recent years, several researchers have adapted Adomian decomposition method (ADM) to solve many kinds of functional equations, which was developed by Adomian in 1980. In [Adomian 1988; 1990], Adomian provided a review of decomposition method in applied mathematics. The solution in this method is considered as the summation of an infinite convergent series without using any restrictive assumptions. A theoretical foundation of Adomian method was developed in [Gabet 1994], Venkatarangan and Rajalakshmi [Venkatarangan and Rajalakshmi 1995] used modified ADM to solve equations containing radical signs. Adomian polynomials are modified by Adomian and Rach in [Adomian and Rach 1996], Luo et. al [Luo et al. 2006] studied the partial solutions on ADM for solving heat and wave equations, Hashim [Hashim 2006] applied ADM to solve linear and nonlinear boundary value problems for fourth order integro-differential equations. In [Hosseini 2006], Hosseini modified the Adomian decomposition method by expressing the source function $f(x)$ in Chebyshev polynomials and solved the nonlinear differential algebraic equations. The ADM is used to solve nonlinear Sturm-Liouville problems in [Somali and Gokmen 2007], Marwat and Asghar [Marwat and Asghar 2008] suggested a two step Adomian decomposition method for solving heat equation with variable coefficients, Liu [Liu 2009] employed Legendre polynomials to improve the Adomian decomposition method and concluded that Chebyshev and Legendre polynomials can be successfully used for ADM and comparatively Chebyshev 
expansion provides the better estimation. The interested reader can see the other applications and modifications of this method in [Ghazanfari and Sepahvandzadeh 2014; Evans et al. 2004; Singh and Kumar 2011; Biazar et al. 2004; Zhang and Lu 2011; Li and Wang 2009; Biazar et al. 2010; Abassy 2010; Bildik and Deniz 2015; Babolian and Biazar 2002].

Further, Khuri [Khuri 2001] developed Laplace Adomian decomposition method and applied to find the solutions of nonlinear differential equations. This method is the combination of two powerful tools, Laplace transform and Adomian decomposition method, which is used to solve extinct functional equations [Wazwaz 2010; Doan 2012]. Hence, there are numerous applications where Laplace Adomian decomposition method is used. The method is also improved and modified from different aspects by some authors [Manafianheris 2012; Kumar et al. 2014].

In this work, our aim is to modify Laplace Adomian decomposition method based on Bernstein polynomials. At the beginning of our technique, we expand the source function, i.e. $f(x)$ as Bernstein polynomials which approximate the function uniformly and then Laplace Adomian decomposition method is applied to solve Volterra integral and integro-differential equations, that gives the tremendous improved results as shown in examples. To the best of our knowledge, Bernstein polynomials is not combined with the LADM. Therefore, this is the new idea which we have used.

\subsection{Bernstein polynomials}

The Berstein basis polynomials which are named after Russian mathematician Sergei Bernstein, is used to approximate the functions and curves. Following are some basic definitions [Quain et al. 2011]:

DEFINITION 1.1 (Bernstein basis polynomials). The Bernstein basis polynomials of degree $n$ form a complete basis over the interval $[0,1]$ and are defined by

$$
B_{k, n}(x)=\left(\begin{array}{l}
n \\
k
\end{array}\right) x^{k}(1-x)^{n-k}, k=0,1,2 \ldots n .
$$

DEFINITION 1.2 (Bernstein polynomials). A linear combination of Bernstein basis polynomials 


$$
B_{n}(x)=\sum_{k=0}^{n} \beta_{k} B_{k, n}(x)
$$

is called the Bernstein polynomial of degree $n$ where $\beta_{k}$ are Bernstein coefficients.

DEFINITION 1.3. With $f$ a real valued function defined and bounded on $[0,1]$, let $B_{n}(f)$ be the polynomial on $[0,1]$, that assigns to $f(x)$ the value

$$
B_{n}(f)=\sum_{k=0}^{n}\left(\begin{array}{l}
n \\
k
\end{array}\right) x^{k}(1-x)^{n-k} f\left(\frac{k}{n}\right)
$$

where $B_{n}(f)$ is the nth Bernstein polynomial for $f(x)$.

The utilizations and properties of Bernstein polynomials have gained much importance in the domain of applied mathematics, physics and computer aided-geometric designs [Farouki 2012; Farouki and Rajan 1998; Bohm et al. 1984; Bhatti and Bracken 2007]. Bernstein polynomials are the basis of approximation theory, with the help of these polynomials Weierstrass approximation theorem [Quain et al. 2011] is proved, which is given as follows:

THEOREM 1.4. For all functions $f$ in $C[0,1]$, the sequence of $B_{n}(f)$ converges uniformly to $f$, where $B_{n}(f)$ is defined by (5).

Using Taylors series, if we approximate a function, curve or surface, it seems that it converges slowly and does not converge to original function. Comparatively, Bernstein polynomials are better approximation to a function. It also has some applications in optimal control theory, stochastic dynamics and in the modelling of chemical reactions [Yousefi and Behroozifar 2010]. Problems like, elliptic and hyperbolic partial differential equations have been solved using Bernstein polynomials by implementation of Galerkin and collocation approaches to determine the coefficients.

The contents of this paper are as follows: in Section 2, we will give analysis of modified LADM; Section 3 gives the convergence analysis of the method; in Section 4 we will give three examples to demonstrate the applicability of the proposed approach. In the last section, conclusions are drawn. 


\section{MODIFIED LAPLACE ADOMIAN DECOMPOSITION METHOD BASED ON BERNSTEIN POLYNOMIALS}

In this section, we are analyzing the method developed in [Rani and Mishra 2017] for nonlinear Volterra integral equation with the difference kernel, i.e $k(x, t)=k(x-t)$ given by (1).

Adopting the standard Laplace Adomian decomposition method, firstly applying Laplace transform on both sides of (1) and with the use of linear property and convolution theorem of Laplace transform, we get

$$
L[u(x)]=L[f(x)]+L[k(x-t)] L[F(u(x))]
$$

According to the LADM technique $u(x)$ can be written as an infinite series given by

$$
u(x)=\sum_{n=0}^{\infty} u_{n}(x)
$$

Then writing the nonlinear term $F(u(x))$ as

$$
F(u(x))=\sum_{n=0}^{\infty} A_{n}(x)
$$

where $A_{n}$ 's are the Adomian polynomials, given by the formula

$$
A_{n}=\frac{1}{n !} \frac{d^{n}}{d \lambda^{n}}\left[f\left(\sum_{i=0}^{n} \lambda^{i} u_{i}\right)\right]_{\lambda=0}
$$

Substituting (7) and (8) into (6), we get

$$
L\left[\sum_{n=0}^{\infty} u_{n}(x)\right]=L[f(x)]+L[k(x-t)] L\left[\sum_{n=0}^{\infty} A_{n}(x)\right]
$$

The linearity property of Laplace transform implies

$$
\sum_{n=0}^{\infty} L\left[u_{n}(x)\right]=L[f(x)]+L[k(x-t)] \sum_{n=0}^{\infty} L\left[A_{n}(x)\right]
$$

Now we are modifying the standard LADM, where the source term is expanded or written in the form of Bernstein polynomials with degree $m$ given by (5). Therefore, we attain

$$
\sum_{n=0}^{\infty} L\left[u_{n}(x)\right]=L\left[B_{m} f(x)\right]+L[k(x-t)] \sum_{n=0}^{\infty} L\left[A_{n}(x)\right]
$$


$u(x)$ can be found by defining the following iterative scheme:

$$
L\left[u_{0}(x)\right]=L\left[B_{m}(f(x))\right]
$$

Taking inverse Laplace transform on both sides of (12), we obtain

$$
u_{0}(x)=L^{-1}\left[L\left(B_{m}(f(x))\right)\right]
$$

Therefore, the initial approximation depends on the Bernstein polynomials of source function, which plays a significant role in the next approximations, hence in the approximate solution of the given problem.

Similarly, we have the general relation as

$$
L\left[u_{n+1}(x)\right]=L[k(x-t)] L\left[A_{n}(x)\right]
$$

For determining the terms $u_{1}, u_{2}, u_{3} \ldots$ of infinite series we use the inverse Laplace transform to above recursive relation and $u(x)$, the approximate solution to given nonlinear Volterra integral equation can be calculated. The same process is used to solve nonlinear Volterra integro-differential equations of the first and second kinds.

The efficacy of technique is demonstrated by convergence analysis and following numerical examples.

\section{CONVERGENCE ANALYSIS}

The convergence analysis is presented here which demonstrate the efficiency of the above-modified technique. Considering $E=(C[J],\|\|$.$) the Banach space of all$ continuous functions on $J$, suppose that there exist a constant $N$ such that $|k(x, t)| \leq N$, for all $(x, t) \varepsilon[0, T]^{2}$.

Also, we suppose that the nonlinear term satisfy the Lipschitz condition, the approximate solution of (1) by using Bernstein polynomials based MLADM, converges to the exact one if $0<\alpha<1$, where $\alpha=N L x$.

Let $U$ be the exact solution and $U^{*}$ be the approximate solution of (1) by taking $n$ terms, then

$$
\begin{array}{r}
\left\|U-U^{*}\right\|=\max _{x \varepsilon J}\left|f(x)+\int_{0}^{x} k(x, t) F(U(t)) d t-B_{m}(f(x))-\int_{0}^{x} k(x, t) F\left(U^{*}(t)\right) d t\right| \\
=\left|f(x)-B_{m}(f(x))\right|+\left|\int_{0}^{x} k(x, t)\left(F(U(t))-F\left(U^{*}(t)\right)\right) d t\right|
\end{array}
$$


Now using the convergence theorem of Bernstein polynomials (1.4) and above given conditions in the statement, we get

$$
\begin{gathered}
\left\|U-U^{*}\right\| \leq \varepsilon+\int_{0}^{x}|k(x, t)|\left|F(U(t))-F\left(U^{*}(t)\right)\right| d t \\
\leq \varepsilon+\int_{0}^{x} N L\left|(U(t))-\left(U^{*}(t)\right)\right| d t \\
\leq \varepsilon+N L x \max _{x \varepsilon J}\left|U(t)-U^{*}(t)\right| \\
\leq \alpha\left\|U-U^{*}\right\|
\end{gathered}
$$

Therefore, if $0<\alpha<1, \alpha=N L x$, the approximate solution converges to exact solution as $n \rightarrow \infty$.

\section{NUMERICAL EXAMPLES}

EXAMPLE 4.1. Consider the following nonlinear Volterra integro-differential equation of the second kind [Wazwaz, 2010]

$$
u^{\prime}(x)=-2 \sin x-\frac{1}{3} \cos x-\frac{2}{3} \cos 2 x+\int_{0}^{x} \cos (x-t) u^{2}(t) d t
$$

with initial condition $u(0)=1$, having the exact solution as $u(x)=\cos x-\sin x$.

In this example, the source term, i.e. $f(x)=-2 \sin x-\frac{1}{3} \cos x-\frac{2}{3} \cos 2 x$. Now using the above technique, we expand $f(x)$ in the terms of Bernstein polynomials of order $m=6$

$$
\begin{array}{r}
f(x) \approx 0.000507191 x^{6}+0.010605381 x^{5}-0.10640906 x^{4}-0.06228815 x^{3}+ \\
1.314840965 x^{2}-1.742867841 x-1
\end{array}
$$

By applying Laplace transform to both sides of (16), we get

$$
L[u(x)]=\frac{1}{s}+\frac{1}{s} L[f(x)]+\frac{1}{s^{2}+1} L\left[u^{2}(x)\right]
$$

The methodology consisting of letting the solution as an infinite series as mentioned above, we have

$$
L\left[\sum_{n=0}^{\infty} u_{n}(x)\right]=\frac{1}{s}+\frac{1}{s} L[f(x)]+\frac{1}{s^{2}+1} L\left[\sum_{n=0}^{\infty} A_{n}(x)\right]
$$



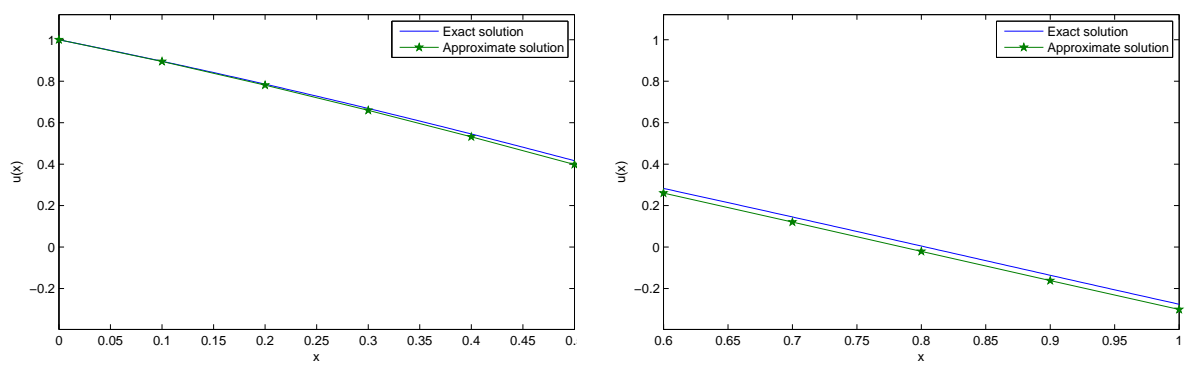

Fig. 1. Comparison of solutions in $[0,0.5]$ and $[0.6,1]$

where the nonlinear term $F(u(x))=u^{2}(x)$ is decomposed in Adomian polynomials, few terms are as follows:

$$
\begin{gathered}
A_{0}=u_{0}^{2} \\
A_{1}=2 u_{0} u_{1} \\
A_{2}=2 u_{0} u_{2}+u_{1}^{2} \\
A_{3}=2 u_{0} u_{3}+2 u_{1} u_{2}
\end{gathered}
$$

The recursive relation is obtained by comparing the terms in (19), which gives

$$
L\left[u_{0}(x)\right]=\frac{1}{s}+\frac{1}{s} L[f(x)]
$$

In general

$$
L\left[u_{n+1}(x)\right]=\frac{1}{s^{2}+1} L\left[A_{n}(x)\right]
$$

Employing the inverse Laplace transform on both sides of (20) and using (17), we get the value of $u_{0}(x)$.

Similarly (21) gives the values of $u_{1}(x), u_{2}(x)$ and so on. Subsequently, one can compare the results from Figure 1, which shows that the approximate solutions are very much close to exact in the interval $[0,0.5]$ than in the interval $[0.6,1]$. 
EXAMPLE 4.2. The following nonlinear Volterra integro-differential equation of the first kind [Wazwaz 2010]

$$
\int_{0}^{x}(x-t) u^{2}(t) d t+\int_{0}^{x}(x-t) u^{\prime \prime}(t) d t=-\frac{15}{32}+\frac{3 x^{2}}{4}+\frac{1}{2} \cos 2 x-\frac{1}{32} \cos 4 x,
$$

with initial condition $u(0)=2, u^{\prime}(0)=0$ which has the exact solution as $u(x)=1+\cos 2 x$.

Apply Laplace transform to both sides of (22) and using the derivative property and convolution theorem, we get

$$
L\left[\int_{0}^{x}(x-t) u^{2}(t) d t\right]+L\left[\int_{0}^{x}(x-t) u^{\prime \prime}(t) d t\right]=L[f(x)]
$$

By solving, we get

$$
\begin{aligned}
& \frac{1}{s^{2}} L\left[u^{2}(x)\right]+L[u(x)]-\frac{2}{s}=L[f(x)] \\
& L[u(x)]=\frac{2}{s}+L[f(x)]-\frac{1}{s^{2}} L\left[u^{2}(x)\right]
\end{aligned}
$$

where the nonlinear term $F(u)=u^{2}$ is decomposed as in the previous example Now proceeding as before, following iterative scheme is obtained:

$$
L\left[u_{0}(x)\right]=\frac{2}{s}+L[f(x)]
$$

In general

$$
L\left[u_{n+1}(x)\right]=-\frac{1}{s^{2}} L\left[A_{n}(x)\right]
$$

Here $f(x)=\frac{-15}{32}+\frac{3 x^{2}}{4}+\frac{1}{2} \cos 2 x-\frac{1}{32} \cos 4 x$ is the source term.

By adopting the above method, we expand $f(x)$ as the Bernstein polynomials:

$$
\begin{array}{r}
f(x) \approx-0.001381627 x^{6}+0.013467998 x^{5}+0.051210391 x^{4}+0.02773726 x^{3}+ \\
0.002551943 x^{2}+0.00001698 x
\end{array}
$$

Applying inverse Laplace transform on both sides of (24), (25) and using the Bernstein polynomials given by (26), we get the values of $u_{0}(x), u_{1}(x), u_{2}(x) \ldots$ Therefore, we find the approximate solution. The approximate solution provides the accurate result or close to the exact solution in very few iterations that is shown in Figure 2. 

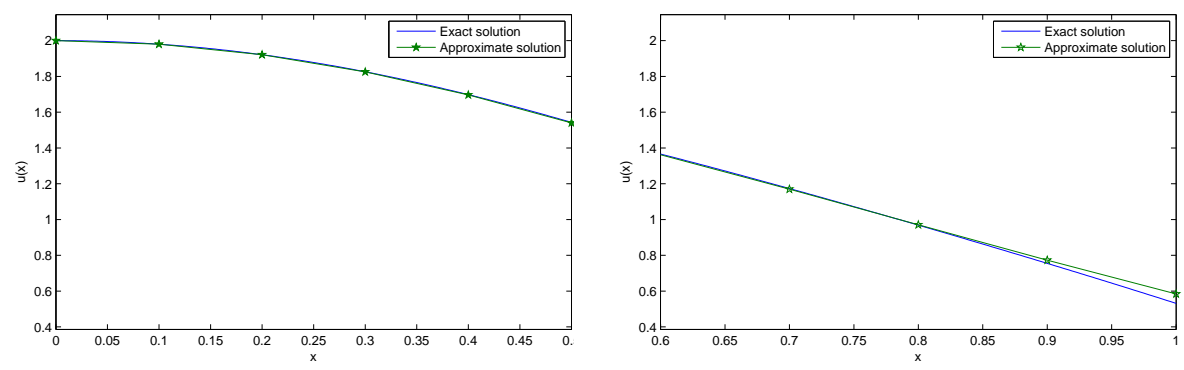

Fig. 2. Comparison of solutions in $[0,0.5]$ and $[0.6,1]$

EXAMPLE 4.3. The nonlinear Volterra integral equation is given by

$$
u(x)=\frac{1}{4}+\frac{x}{2}+e^{x}-\frac{e^{2 x}}{4}+\int_{0}^{x}(x-t) u^{2}(t) d t,
$$

having the exact solution as $u(x)=e^{x}$.

The source term in (27) is $f(x)=\frac{1}{4}+\frac{x}{2}+e^{x}-\frac{e^{2 x}}{4}$ which can be expanded in the Bernstein polynomials, here taking $m=10$.

$$
\begin{aligned}
& f(x) \approx-0.000000056 x^{10}-0.00000332 x^{9}-0.00006387 x^{8}-0.00076576 x^{7}-0.00589954 x^{6} \\
& -0.03027348 x^{5}-0.1004593 x^{4}-0.18599482 x^{3}-0.05372434 x^{2}+0.99820229 x+1
\end{aligned}
$$

Taking Laplace transform on both sides of (27), gives

$$
L[u(x)]=L[f(x)]+\frac{1}{s^{2}} L\left[u^{2}(x)\right]
$$

Now $u(x)$ can be evaluated based on Bernstein polynomials of $f(x)$ and with decomposing the nonlinear term in Adomian polynomials, which implies the relation

$$
L\left[u_{0}(x)\right]=L[f(x)]
$$

In general

$$
L\left[u_{n+1}(x)\right]=\frac{1}{s^{2}} L\left[A_{n}(x)\right]
$$

Substituting the approximated value of $f(x)$ from (28) in (30) and having inverse Laplace transform on both sides of (30), (31) give the values of $u_{0}(x), u_{1}(x), u_{2}(x), \ldots u_{n}(x)$. The sum of these terms will yield the value of truncated sum of $u(x)$. It is found that the error between exact and approximate solution is very less as shown in Figure 3 and reveals that the Bernstein polynomials based modification of LADM gives the solution in good agreement. 

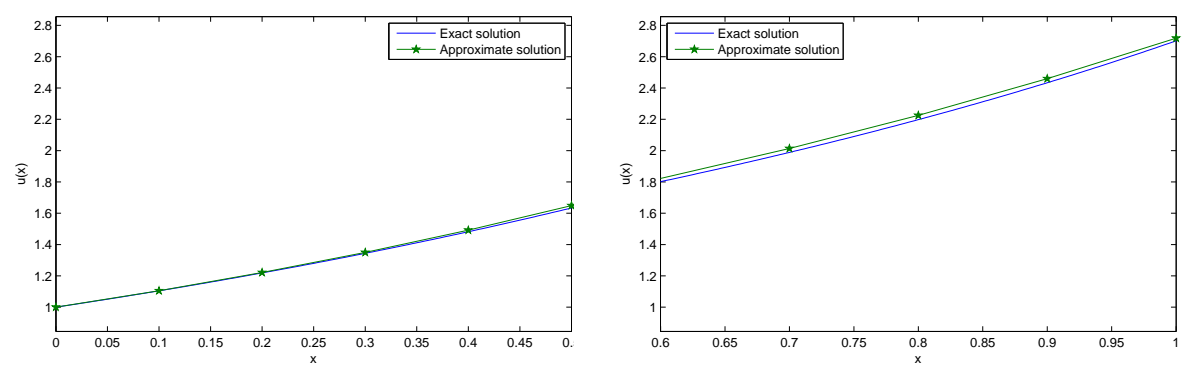

Fig. 3. Comparison of solutions in $[0,0.5]$ and $[0.6,1]$

Table I. Comparison of approximate solution by proposed method with exact solution of examples

\begin{tabular}{cllllll}
\hline & \multicolumn{2}{c}{ Example1 } & \multicolumn{2}{c}{ Example2 } & \multicolumn{2}{c}{ Example3 } \\
$\mathrm{x}$ & Exact & Approximate & Exact & Approximate & Exact & Approximate \\
\hline 0 & 1 & 1 & 2 & 2 & 1 & 1 \\
0.1 & 0.8952 & 0.8964 & 1.9801 & 1.9801 & 1.1052 & 1.1044 \\
0.2 & 0.7814 & 0.7858 & 1.9211 & 1.9212 & 1.2214 & 1.2188 \\
0.3 & 0.6598 & 0.6687 & 1.8253 & 1.8259 & 1.3499 & 1.3441 \\
0.4 & 0.5316 & 0.5455 & 1.6967 & 1.6982 & 1.4918 & 1.4819 \\
0.5 & 0.3982 & 0.4166 & 1.5403 & 1.5431 & 1.6487 & 1.6338 \\
0.6 & 0.2607 & 0.2828 & 1.3624 & 1.3663 & 1.8221 & 1.8018 \\
0.7 & 0.1206 & 0.1451 & 1.1700 & 1.1734 & 2.0138 & 1.9887 \\
0.8 & -0.0206 & 0.0049 & 0.9708 & 0.9687 & 2.2255 & 2.1978 \\
0.9 & -0.1617 & -0.1361 & 0.7728 & 0.7550 & 2.4596 & 2.4335 \\
1 & -0.3012 & -0.2758 & 0.5839 & 0.5314 & 2.7183 & 2.7014 \\
\hline
\end{tabular}

The numerical results by using modified LADM based on Bernstein polynomials are also presented in Table I, which shows the performance of proposed technique.

\section{CONCLUSIONS}

For solving nonlinear Volterra integral and integro-differential equations a modification in standard Laplace Adomian decomposition method based on Bernstein polynomials is used here. Comparisons and analyses conclude that not only the orthogonal polynomials like Legendre, Chebyshev or Jacobi polynomials can improve the ADM, the Bernstein polynomials can also improve the source term as it is the better approximation to a function and hence the approximate solution converges to exact one as shown in the examples. 


\section{REFERENCES}

ABASSY, T. A. 2010. Improved Adomian decomposition method. Computers and Mathematics with Applications 59, 42-54.

Adomian, G. 1988. A review of the decomposition method in applied mathematics. Journal of Mathematical Analysis and Applications 135, 501-544.

Adomian, G. 1990. A review of the decomposition method and some recent results for nonlinear equations. Mathematics Computer and Modelling 13, 17-43.

Adomian, G. AND RACH, R. 1996. Modified Adomian polynomials. Mathematics and Computer Modelling 24, 39-46.

BABOlian, E. AND BIAZAR, J. 2002. Solution of nonlinear equations by modified Adomian decomposition method. Applied Mathematics and Computation 132, 167-172.

Bahuguna, D., Ujlayan, A., And Pandey, D. N. 2009. A comparative study of numerical methods for solving an integro-differential equation. Computers and Mathematics with Applications 57, 1485 1493.

Bhatti, M.I. And BRACKEn, P. 2007. Solutions of differential equations in a Bernstein polynomial basis. Journal of Computational and Applied Mathematics 205, 272-280.

Biazar, J., Babolian, E., AND Islam, R. 2004. Solution of the system of ordinary differential equations by Adomian decomposition method. Applied Mathematics and Computation 147, 713-719.

BiAzAr, J., PorshokUhi, M. G., AND GHAnBARI, B. 2010. Extracting a general iterative method from an Adomian decomposition method and comparing it to the variational iteration method. Computers and Mathematics with Applications 59, 622-628.

BIldik, N. AND DENIZ, S. 2015. Modified Adomian decomposition method for solving Riccati differential equations. Review of the Air Force Academy 30, 21-26.

Bohm, W., Farin, G., And Kahmann, J. 1984. A survey of curve and surface methods in cagd. Computer Aided Geometric Design 1, 1-60.

DoAn, N. 2012. Solution of the system of ordinary differential equations by combined Laplace transform Adomian decomposition method. Mathematical and Computational Applications 17, 203-211.

Evans, D.J. AND Raslan, K. R. 2004. The Adomian decomposition method for solving delay differential equation. International Journal of Computer Mathematics 00, 1-6.

FAROUKI, R. T. 2012. The Bernstein polynomial basis: A centennial retrospective. Computer Aided Geometric Design 29, 379-419.

FAROUKi, R. T. AND RAJAN, V. T. 1998. Algorithms for polynomials in Bernstein form. Computer Aided Geometric Design 5, 1-26.

GaBET, L. 1994. The theoretical foundation of the Adomian method. Computers and Mathematics with Applications 27, 41-52.

Ghazanfari, B. And Sepahvandzadeh, A. 2014. Adomian decomposition method for solving Bratu's type equation. Journal of Mathematics and Computer Science 8, 236-244.

HASHIM, I. 2006. Adomian decomposition method for solving BVPs for fourth-order integro-differential equations. Journal of Computational and Applied Mathematics 193, 658-664.

Hosseini, M. M. 2006. Adomian decomposition method for solution of nonlinear differential algebraic equations. Applied Mathematics and Computation 181, 1737-1744.

KHURI, S. A. 2001. A Laplace decomposition algorithm applied to a class of nonlinear differential equation. Journal of Applied Mathematics 1, 141-155. 
Kumar, S., Kumar, D., Abbasbandy, S., And Rashidi, M. M. 2014. Analytic solution of fractional Navier-Stokes equation by using modified Laplace decomposition method. Ain Shams Engineering Journal 5, 569-574.

LI, C. AND WANG, Y. 2009. Numerical algorithm based on Adomian decomposition for fractional differential equations. Computers and Mathematics with Applications 57, 1672-1681.

LIU, Y. 2009. Adomian decomposition method with orthogonal polynomials:Legendre polynomials. Mathematical and Computer Modelling 49, 1268-1273.

LuO, X.-G., WU, Q.-B., AND ZHANG, B.-Q. 2006. Revisit on partial solutions in the Adomian decomposition method: solving heat and wave equations. Journal of Mathematical Analyis and Applications 321, 353-363.

Maleknejad, K., Hashemizadeh, E., and Ezzati, R. 2011. A new approach to the numerical solution of Volterra integral equations by using Bernsteins approximation. Communication in Nonlinear Science and Numerical Simulation 16, 647-655.

MALEKNEJAD, K. AND NAJAFI, E. 2011. Numerical solution of nonlinear Volterra integral equations using the idea of quasilinearization. Communication in Nonlinear Science and Numerical Simulation 20, 93-100.

MANAFIANHERIS, J. 2012. Solving the integro-differential equations using the modified Laplace Adomian decomposition method. Journal of Mathematical Extension 6, 41-55

Marwat, D. N. K. AND Asghar, S. 2008. Solution of the heat equation with variable properties by two-step Adomian decomposition method. Mathematical and Computer Modelling 48, 83-90.

Quain, W., Riedel, M. D., AND Rosenberg, I. 2011. Uniform approximation and Bernstein polynomial with coefficients in the unit interval. European Journal of Combinatorics 32, 448-463.

Singh, N. AND KumAR, M. 2011. Adomian decomposition method for solving higher order boundary value problems. Mathematical Theory and Modeling 2, 11-22.

Somali, S. And Gokmen, G. 2007. Adomian decomposition method for nonlinear Sturm-Liouville problems. Surveys in Mathematics and its Applications 2, 11-20.

Venkatarangan, S. N. And Rajalakshmi, K. 1995. Modification of Adomian's decomposition method to solve equations containing radicals. Computers and Mathematics with Applications 29, 75 80.

WAZWAZ, A. M. 2010. The combined Laplace transform-Adomian demcomposition method for handling nonlinear Volterra integro-differential equations. Applied Mathematics and Computation 216, 1304 1309.

WAZWAZ, A. M. 2011. Linear and Nonlinear Integral Equations: Methods and Applications. Springer.

Yousefi, S. A. AND BehroozIFAR, M. 2010. Operational matrices of Bernstein polynomials and their applications. International Journal of System Science 41, 709-716.

ZHANG, B. AND LU, J. 2011. Exact solutions of homogenous partial differential equations by a new Adomian decomposition method. Procedia Environmental Science 11, 440-446.

RANI, D. AND MishrA, V. 2017. Approximate solution of boundary value problem with Bernstein polynomial Laplace decomposition method. International Journal of Pure and Applied Mathematics $114,823-833$. 


\section{Dimple Rani}

Department of Mathematics,

Sant Longowal Institute of Engineering and Technology,

Longowal-148106 (Punjab), India

email: chawla23dimple@gmail.com

\section{Vinod Mishra}

Department of Mathematics,

Sant Longowal Institute of Engineering and Technology,

Longowal-148106 (Punjab), India

email: vinodmishra.2011@rediffmail.com 